\title{
THE LINES OF PRINCIPAL STRESS IN THE PLANE PROBLEM OF PLASTICITY*
}

\author{
By W. S. AMENT (Brown University)
}

Consider a state of plane strain in an incompressible plastic body yielding under a constant maximum shearing stress. J. Boussinesq ${ }^{1}$ has shown that the lines of principal stress then form an "equiareal pattern," i.e. from the two families of lines of principal stress individuals can be selected so as to render equal in area the meshes formed by these lines. In a recent paper ${ }^{2} M$. A. Sadowsky has stressed the importance of this result and has coined the term "equiareal pattern." The present note aims at establishing the relation between Boussinesq's result and a theorem concerning the lines of principal curvature on certain Weingarten surfaces.

If the lines of curvature are chosen as parametric curves and $\kappa$ and $\kappa^{\prime}$ denote the principal curvatures corresponding to the directions of $v=$ const. and $u=$ const. respectively, the Mainardi-Codazzi relations take the form ${ }^{3}$

$$
\begin{aligned}
& \frac{\partial}{\partial u}(\log G)=\frac{2}{\kappa-\kappa^{\prime}} \frac{\partial \kappa^{\prime}}{\partial u}, \\
& \frac{\partial}{\partial v}(\log E)=-\frac{2}{\kappa-\kappa^{\prime}} \frac{\partial \kappa}{\partial v} .
\end{aligned}
$$

Consider now the Weingarten surfaces for which the difference of the principal curvatures has a constant value. Elimination of $\kappa$ and $\kappa^{\prime}$ between the equations (1) then leads to

$$
\frac{\partial^{2}}{\partial u \partial v}(\log E G)=0
$$

Hence $E G=f(u) g(v)$. A transformation of the type $\bar{u}=\bar{u}(u), \bar{v}=\bar{v}(v)$ only relabels the parametric curves but does not affect their geometric properties. Define $\bar{u}$ and $\bar{v}$ by

$$
\frac{d \bar{u}}{d u}=\sqrt{f(u),} \quad \frac{d \bar{v}}{d v}=\sqrt{g(v) .}
$$

For these new parameters $\bar{E} \bar{G}=1$, i.e. the meshes formed by the parametric curves corresponding to two sets of equidistant values of $\bar{u}$ and $\bar{v}$ are equal in area. The lines of principal curvature on a Weingarten surface with $\kappa-\kappa^{\prime}=$ const. therefore form an equiareal pattern.

The relation between this theorem and Boussinesq's result is immediate. Introduce rectangular Cartesian coordinates $O, x, y, z$, the plane $O, x, y$ having the orientation of the plane of strain. The normal stresses $\sigma_{x}, \sigma_{y}$ and the shearing stress $\tau$ then can be derived from a stress function $F$ according to

* Received Nov. 6, 1942.

1 C. R. Ac. Sci. Paris, 74, 242 (1872).

2 Trans. Am. Soc. Mech. Eng. 63, A-74 (1941).

${ }^{3}$ See for instance C. E. Weatherburn, Differential geometry, vol. II, Cambridge 1930, p. 52. 


$$
\sigma_{x}=\frac{\partial^{2} F}{\partial y^{2}}, \quad \sigma_{y}=\frac{\partial^{2} F}{\partial x^{2}}, \quad \tau=-\frac{\partial^{2} F}{\partial x \partial y} .
$$

The yield condition

$$
\left(\sigma_{x}-\sigma_{y}\right)^{2}+4 r^{2}=\text { const. }
$$

furnishes the following differential equation for $F$ :

$$
\left(\frac{\partial^{2} F}{\partial x^{2}}-\frac{\partial^{2} F}{\partial y^{2}}\right)^{2}+4\left(\frac{\partial^{2} F}{\partial x \partial y}\right)^{2}=\text { const. }
$$

Consider the surface $S$ defined by $z(x, y)=\alpha F(x, y)$, where $\alpha$ is an arbitrary small constant rendering small the slope which the tangential planes of $S$ have with respect to the plane $O, x, y$. If terms of the second order in $\partial F / \partial x$ and $\partial F / \partial y$ are neglected, the difference of the principal curvatures of $S$ is seen to equal the square root of the left hand side of (2) multiplied by $\alpha$. The surface $S$ therefore is a Weingarten surface of the type considered above. The orthogonal projections of its lines of principal curvature on the plane $O, x, y$ are the lines of principal stress. 